\title{
Espessura do músculo adutor do polegar em idosos hospitalizados: relação com sexo e idade
}

\author{
Adductor pollicis muscle thickness in hospitalized elderly patients: relation with sex and age
}

\author{
Raquel Milani El Kika , Janaína Raasch ${ }^{b}$, Melissa Côrtes da Rosac, Irenio Gomes ${ }^{\mathrm{d}}$, \\ Carla Helena Augustin Schwanke \\ a Nutricionista. Doutoranda do Programa de Pós-Graduação em Gerontologia Biomédica do Instituto de Geriatria e Gerontologia da Pontifícia Universidade Católica \\ do Rio Grande do Sul (IGG-PUCRS). \\ ${ }^{\mathrm{b}}$ Nutricionista. \\ c Nutricionista. Doutoranda do Programa de Pós-Graduação em Gerontologia Biomédica do IGG-PUCRS. \\ d Médico. Doutor em Medicina. Pós-doutor no Centre Hospitalier Universitaire de Bicêtre, França. \\ e Médica. Doutora em Gerontologia Biomédica pelo IGG-PUCRS.
}

RESUMO

Objetivo: Relacionar a espessura do músculo adutor do polegar de idosos hospitalizados com sexo e idade.

Materiais e Métodos: Foi realizado um estudo transversal, de maio de 2014 a abril de 2015, com uma amostra de conveniência de 119 idosos hospitalizados em uma unidade geriátrica de um hospital universitário de Porto AlegreRS, Brasil. A espessura do músculo adutor do polegar foi aferida na mão dominante e na mão não dominante, com um Plicômetro Lange ${ }^{\circledR}$. Foram utilizados os testes t de Student para dados pareados e t de Student para dados independentes.

Resultados: A média da idade foi $82,3 \pm 8,1$ anos (60 a 97 anos). A maioria dos pacientes era do sexo feminino $(62,2 \%)$. A média da espessura do músculo adutor do polegar na amostra total foi $14,96 \pm 4,62 \mathrm{~mm}$ e não foi verificada diferença significativa entre as mãos dominante e não dominante. Os homens apresentaram valores médios significativamente maiores que as mulheres em ambas as mãos (Dominante - homens: 16,79 $\pm 4,21 \mathrm{~mm}$,


correlação inversa e fraca da espessura do músculo adutor do polegar com idade em ambas as mãos (Dominante $r=-0,300$; Não dominante $r=-0,308)$. Também foi verificada diferença significativa entre as médias da espessura do músculo adutor do polegar e faixa etária em ambas as mãos (Dominante - 60-79 anos: 16,63 $\pm 4,25 \mathrm{~mm}$, $\geq 80$ anos: 14,27 $\pm 4,47 \mathrm{~mm}$; Não dominante $-60-79$ anos: $16,47 \pm 3,94 \mathrm{~mm}, \geq 80$ anos: $14,10 \pm 4,90 \mathrm{~mm})$.

Conclusão: Em idosos hospitalizados, a espessura do músculo adutor do polegar apresentou-se maior nos indivíduos mais jovens (60-79 anos) e nos do sexo masculino.

Palavras-chave: idoso; desnutrição; antropometria; avaliação nutricional.

ABSTRACT

Objective: To relate the adductor pollicis muscle thickness of hospitalized elderly patients to sex and age.

Materials and Methods: A cross-sectional study was conducted, from May 2014 through April 2015, with a convenience sample of 119 elderly patients, hospitalized in a geriatric unit of a university hospital of Porto Alegre-RS, Brazil. The adductor pollicis muscle thickness values of the dominant and non-dominant hand were measured using a Lange ${ }^{\circledR}$ skinfold caliper. Student $t$-tests were used for both paired and independent data.

Results: The mean age was $82.3 \pm 8.1$ years (60 to 97 years) and the majority of the patients were female (62.2\%). The mean adductor pollicis muscle thickness value in the total sample was $14.96 \pm 4.62 \mathrm{~mm}$ and no significant difference was verified between dominant and non-dominant hand. Men presented significantly higher mean values than women in both hands (Dominant - men: $16.79 \pm 4.21 \mathrm{~mm}$; women: $13.99 \pm 4.39 \mathrm{~mm}$ : Non-dominant - men: $16.58 \pm 4.13 \mathrm{~mm}$; women: $13.84 \pm 4.78 \mathrm{~mm})$. A weak inverse correlation was observed between the adductor pollicis muscle thickness and age in both hands (Dominant $-r=-0.300$; Nondominant $-r=-0.308$ ). It was also verified a significant difference between the means of the adductor pollicis muscle thickness and age group in both hands (Dominant: $60-79$ years: $16.63 \pm 4.25 \mathrm{~mm}$; $\geq 80$ years: $14.27 \pm 4.47 \mathrm{~mm}$; Non-dominant $-60-79$ years: $16.47 \pm 394 \mathrm{~mm} ; \geq 80$ years: $14.10 \pm 4.90 \mathrm{~mm})$.

Conclusion: In hospitalized elderly patients, the adductor pollicis muscle thickness was greater among younger individuals (60-79 years) and in males.

Keywords: elderly; malnutrition; anthropometry; nutritional assessment.

\section{Correspondência:}

Carla Helena Augustin SCHWANKE

Av. Ipiranga, 6681, Prédio 81, 7ㅇaa andar, sala 703

90619-900 Porto Alegre, RS, Brasil

E-mail: schwanke@pucrs.br 


\section{INTRODUÇÃO}

A desnutrição é frequente entre idosos hospitalizados ${ }^{1-4}$. Ter 60 anos ou mais é um dos fatores associados à maior frequência de desnutrição hospitalar. No Brasil, o Inquérito Brasileiro de Avaliação Nutricional (IBRANUTRI), realizado com 4000 indivíduos hospitalizados em 12 Estados brasileiros e no Distrito Federal, constatou uma prevalência de 52,8\% de desnutrição entre os idosos ${ }^{1}$.

Entre as principais causas de desnutrição em idosos estão as alterações biológicas e psicossociais, o número elevado de doenças associadas e uso de medicações ${ }^{5}$. As consequências da desnutrição são: o aumento do risco de morbidade e mortalidade, do tempo de internação, de readmissões hospitalares e de redução da capacidade funcionallo-9. Para identificar a desnutrição em idosos hospitalizados é necessário realizar uma avaliação nutricional, compreendendo anamnese e exame físico ${ }^{10}$, ou de forma rápida, com a aplicação de instrumentos de triagem nutricional ${ }^{6,11}$ como o Nutritional Risk Screening (NRS 2002) e a Mini Avaliação Nutricional $\left(M N A^{\circledR}\right)^{10,12-14}$. Outro instrumento de triagem nutricional descrito na literatura é a força de preensão palmar (FPP) medida por dinamometria ${ }^{15-18}$. Mais recentemente, a avaliação da espessura do músculo adutor do polegar (EMAP) tem sido apontada como uma alternativa, por ser não invasiva, de rápida obtenção e de baixo custo ${ }^{19}$. Esse músculo é o único que permite a medida direta de sua espessura ${ }^{20}$ e é responsável pela força de pinçamento do $\operatorname{polegar}^{21}$. A EMAP foi descrita na avaliação de indivíduos saudáveis e em diversas situações clínicas ${ }^{19}$ e valores diminuídos têm sido relacionados ao aumento no tempo de internação e complicações infecciosas ${ }^{22}$, reforçando sua relação com a desnutrição.

Pereira et al. ${ }^{19}$, em uma revisão integrativa da literatura, encontraram 10 artigos publicados nos últimos 10 anos sobre EMAP, contudo, estudos em idosos hospitalizados são escassos. Assim, o objetivo deste estudo foi relacionar a EMAP de idosos hospitalizados com sexo e idade.

\section{MATERIAIS E MÉTODOS}

Trata-se de um estudo transversal. Participaram do estudo idosos internados na Unidade Geriátrica de um hospital universitário de Porto Alegre, Rio Grande do Sul, Brasil (PUCRS), no período de maio de 2014 a abril de 2015. A amostragem foi de conveniência, sendo inclusos indivíduos com idade $\geq 60$ anos nos quais foi possível obter a EMAP em ambas as mãos. Foram excluídos pacientes que tivessem qualquer enfermidade que impossibilitasse a aferição da EMAP.
As variáveis em investigação são idade, faixa etária, sexo e medida da EMAP da mão dominante (MD) e da mão não dominante (MND).

As medidas da EMAP da MD e da MND foram realizadas na admissão do paciente, por avaliadores treinados, com o voluntário sentado, com a mão repousando sobre o joelho e cotovelo formando ângulo de noventa graus sobre o membro inferior ${ }^{21}$. Em pacientes acamados, a aferição foi realizada com a mão apoiada no abdômen do paciente, com o cotovelo, formando ângulo de noventa graus $^{23}$. Para tanto, foi utilizado um Plicômetro Lange ${ }^{\circledR}$ e foi exercida pressão contínua de $10 \mathrm{~g} / \mathrm{mm}^{2}$ para pinçar o músculo adutor, no vértice de um ângulo imaginário formado pelo dedo indicador e o polegar da mão ${ }^{21,23}$. Foram realizadas três medidas consecutivas e foi considerado como valor final a média das três mensurações na MD e $\mathrm{MND}^{23}$.

Os dados foram armazenados em banco de dados Excel e analisados por meio do pacote estatístico SPSS 17.0 (SPSS Inc. Chicago IL, USA). A análise descritiva foi realizada através de medidas de frequência, tendência central e dispersão. Foi verificada a concordância intra e interavaliadores pelo Coeficiente de Correlação Intraclasse (ICC), e a concordância foi classificada segundo proposta de Fleiss \& Cohen ${ }^{24}$. As variáveis EMAP da MD e da MND apresentaram curvas de distribuição normal. Para comparar a média das medidas da EMAP em relação ao sexo e faixa etária foi utilizado o teste t de Student e para comparação das medidas da MD com as da MND foi utilizado o teste t para amostras pareadas. Foi utilizado o teste de Correlação de Pearson para analisar a correlação da média da EMAP com a idade. A classificação desse coeficiente foi realizada segundo Callegari-Jacques ${ }^{25}$. Foram considerados significativos os valores de $\mathrm{P}<0,05$.

O projeto guarda-chuva "Força de Preensão Palmar e Espessura do Músculo Adutor do Polegar como Instrumentos de Rastreio do Estado Nutricional, Capacidade Funcional e Mortalidade em Idosos Hospitalizados" foi aprovado pelo Comitê de Ética em Pesquisa da instituição (CAAE: 26825614.7.0000.5336, parecer 636.335 de 29/04/2014). Todos os participantes foram instruídos acerca dos procedimentos que envolveram a pesquisa e assinaram o Termo de Consentimento Livre e Esclarecido.

\section{RESULTADOS}

Foram avaliados 119 indivíduos (60 a 97 anos) com média de idade de $82,26 \pm 8,06$ anos. A maioria era do sexo feminino, da faixa etária $\geq 80$ anos, apresentando média geral do valor da EMAP de 14,96 $\pm 4,62 \mathrm{~mm}$ (Tabela 1). 
Tabela 1. Descrição da medida da espessura do músculo adutor do polegar da mão dominante e não dominante de idosos hospitalizados e análise da associação com sexo e faixa etária.

\begin{tabular}{|c|c|c|c|c|c|c|}
\hline Variáveis & n (\%) & $\begin{array}{c}\text { EMAP MD } \\
\text { Média } \pm \text { DP } \\
(\mathbf{m m})\end{array}$ & $\begin{array}{l}\text { EMAP MND } \\
\text { Média } \pm \text { DP } \\
(\mathbf{m m})\end{array}$ & $\begin{array}{l}\text { Diferença } \\
\text { Média } \pm \text { EP } \\
(\mathbf{m m})\end{array}$ & $\begin{array}{l}\text { Diferença } \\
\text { (IC 95\%) }\end{array}$ & $p^{*}$ \\
\hline \multicolumn{7}{|l|}{ Sexo } \\
\hline Feminino & $74(62,2)$ & $13,99 \pm 4,39$ & $13,84 \pm 4,78$ & $0,15 \pm 0,28$ & $-0,41-0,71$ & 0,598 \\
\hline Masculino & $45(37,8)$ & $16,79 \pm 4,21$ & $16,58 \pm 4,13$ & $0,20 \pm 0,27$ & $-0,36-0,76$ & 0,472 \\
\hline$p$ & & 0,001 & 0,002 & & & \\
\hline \multicolumn{7}{|l|}{ Faixa etária } \\
\hline $60-79$ anos & $39(32,8)$ & $16,63 \pm 4,25$ & $16,47 \pm 3,94$ & $0,16 \pm 0,30$ & $-0,44-0,76$ & 0,588 \\
\hline$\geq 80$ anos & $80(67,2)$ & $14,27 \pm 4,47$ & $14,10 \pm 4,90$ & $0,17 \pm 0,27$ & $-0,36-0,70$ & 0,522 \\
\hline$p$ & & 0,007 & 0,010 & & & \\
\hline Amostra total & $119(100)$ & $15,04 \pm 4,52$ & $14,87 \pm 4,72$ & $0,17 \pm 0,20$ & $-0,23-, 057$ & 0,409 \\
\hline
\end{tabular}

EMAP: espessura do músculo adutor do polegar; MD: mão dominante; MND: mão não dominante; DP: desvio padrão; EP: erro padrão. $p^{*}$ : teste $t$ de Student para dados pareados; $p$ : teste t de Student para dados independentes.

Através do ICC foi verificada excelente concordância intra-avaliadores, tanto para a avaliadora $1(r=0,999$; $p<0,001)$ como para a avaliadora $2(r=0,997 ; p<0,001)$, em uma subamostra de 47 idosos deste estudo. A análise interavaliadores também demonstrou excelente concordância $(r=0,993 ; p<0,001)$.

Na Tabela 1, também são apresentadas a descrição da EMAP da MD e da MND e a análise da associação com sexo e faixa etária, sem ter sido observada diferença significativa nas médias da EMAP da MD e da MND. No entanto, foi verificado maior valor médio da EMAP para os homens, tanto na MD como na MND. Também foi verificada diferença significativa entre as médias da EMAP e faixa etária, em ambas as mãos.

Verificou-se que a EMAP da MD $(r=-0,300 ; p=0,001)$ e da MND ( $r=-0,308 ; p=0,001)$, apresentaram uma correlação fraca e inversa com a idade.

\section{DISCUSSÃO}

No presente estudo, buscou-se descrever a EMAP de idosos hospitalizados e analisar sua associação com variáveis demográficas. Ao melhor do nosso conhecimento, trata-se do primeiro estudo a incluir apenas indivíduos idosos em sua amostra. Pode-se observar que a EMAP era maior nos indivíduos do sexo masculino e nos idosos mais jovens. Ao se realizar uma busca por estudos que avaliaram a EMAP, foram encontrados 12 artigos originais ${ }^{22,23,26-36}$, todos brasileiros. Uma síntese dos principais resultados destes estudos é apresentada na Tabela 2.

Os valores médios para a EMAP observados no presente estudo foram semelhantes às médias encontradas em três estudos, um deles com pacientes clínicos e cirúrgicos hospitalizados ${ }^{23}$, um com pacientes hospitalizados candidatos à procedimento cirúrgico ${ }^{26}$ e outro com pacientes em hemodiálise ${ }^{29}$. Nos dois primeiros estudos, os indivíduos encontravam-se hospitalizados por motivos clínicos e/ou cirúrgicos e, apesar da média de idade dos mesmos ser mais baixa, provavelmente suas condições de saúde também comprometiam a EMAP. Já no terceiro estudo, ressalta-se que os autores apresentaram os valores médios de EMAP nos pacientes com idade abaixo e acima de 60 anos, sendo este o grupo etário com valores semelhantes aos identificados no presente estudo.

Dois estudos, que avaliaram indivíduos mais jovens e na comunidade, identificaram valores médios de EMAP superiores aos do presente estudo. Tal achado ocorreu, provavelmente, em função da menor idade e por se tratarem de indivíduos saudáveis ${ }^{27,28}$.

Surpreendentemente, outros estudos verificaram valores inferiores de EMAP, em adultos e idosos, tanto saudáveis ${ }^{30}$ como em diferentes condições clínicas como câncer ${ }^{10,31}$, insuficiência renal crônica em hemodiálise ${ }^{32,33}$, assim como pacientes hospitalizados clínicos e cirúrgicos ${ }^{34}$ e pacientes cirúrgicos ${ }^{22,35}$. Uma das possíveis explicações para a variação nos valores de EMAP encontrados nos estudos anteriormente citados relaciona-se a sua aferição (instrumento de aferição, ponto anatômico onde foi obtida a medida, variação interavaliadores), que é um aspecto também apontado por Gonzalez et al. ${ }^{27}$. Cabe salientar que, no presente estudo, foi verificada excelente concordância tanto intra-avaliadores, como interavaliadores e que foi utilizado um Plicômetro Lange ${ }^{\circledR}$ para a obtenção das medidas da EMAP.

Também se verificou que valores médios da EMAP podem variar de acordo com sexo. Nos estudos de Gonzalez et al. ${ }^{27}$, Lameu et al. ${ }^{30}$ e Bielemann et al. ${ }^{28}$, apesar de terem sido realizados com indivíduos saudáveis, os valores de EMAP em homens foram maiores do que nas mulheres, conforme verificado em nosso estudo. 
Tabela 2. Estudos de determinação de valores de espessura do músculo adutor do polegar.

\begin{tabular}{|c|c|c|c|}
\hline Autores & Amostra e média de idade & Valores médios de EMAP & Diferença da EMAP entre as mãos, por sexo e idade \\
\hline Caporossi et al. ${ }^{23}$ & $\begin{array}{l}\text { Pacientes clínicos e cirúrgicos } \\
\text { hospitalizados } n=246 \\
\text { Média da idade }=62 \text { anos }\end{array}$ & $\begin{array}{l}\text { Mão direita: } 16,0 \pm 5,8 \mathrm{~mm} \\
\text { Mão esquerda: } 15,0 \pm 5,8 \mathrm{~mm}\end{array}$ & $\begin{array}{l}\text { Foi observada forte correlação }(r=0,84, p<0,001) \text { entre os } \\
\text { valores de EMAP da mão esquerda e da mão direita, embora a } \\
\text { média da EMAP da mão direita tenha sido superior à da mão } \\
\text { esquerda }(p<0,001) \text {. }\end{array}$ \\
\hline Melo et al. ${ }^{26}$ & $\begin{array}{l}\text { Pacientes candidatos a } \\
\text { procedimento cirúrgico } \\
\mathrm{n}=151 \\
\text { Média da idade }=51 \pm 15 \text { anos }\end{array}$ & $\begin{array}{l}\text { MD pacientes eutróficos: } \\
\text { 13,9 } \pm 3,4 \mathrm{~mm} \\
\text { MD pacientes desnutridos: } \\
\text { 10,8 } \pm 2,9 \mathrm{~mm} \\
\text { MND pacientes eutróficos: } \\
\text { 13,1 } \pm 3,7 \mathrm{~mm} \\
\text { MND pacientes desnutridos: } \\
\text { 10,3 } \pm 3,1 \mathrm{~mm}\end{array}$ & $\begin{array}{l}\text { A EMAP foi determinada segundo a classificação do estado } \\
\text { nutricional, de acordo com o índice de massa corporal. A EMAP } \\
\text { da MD mostrou-se superior em relação a da MND. }\end{array}$ \\
\hline Gonzalez et al. ${ }^{27}$ & $\begin{array}{l}\text { Indivíduos saudáveis } \\
\mathrm{n}=300 \\
\text { Média da idade }=44 \pm 18 \text { anos }\end{array}$ & $\begin{array}{l}\text { Amostra total: } \\
\text { MD: } 22,9 \pm 5,0 \mathrm{~mm} \\
\text { MND: } 21,9 \pm 5,0 \mathrm{~mm} \\
\text { Homens: } \\
\text { MD: } 26,1 \pm 4,4 \mathrm{~mm} \\
\text { MND: } 25,1 \pm 4,4 \mathrm{~mm} \\
\text { Mulheres: } \\
\text { MD: } 19,8 \pm 3,3 \mathrm{~mm} \\
\text { MND: } 18,7 \pm 3,1 \mathrm{~mm} \\
\text { Homens > } 60 \text { anos: } \\
\text { MD: } 23,0 \pm 4,5 \mathrm{~mm} \\
\text { Mulheres }>60 \text { anos: } \\
\text { MD: } 17,8 \pm 3,1 \mathrm{~mm}\end{array}$ & $\begin{array}{l}\text { Não foi encontrada diferença significativa entre EMAP na MD e } \\
\text { MND entre o grupo de } 18-30 \text { anos e o grupo com idade } \geq 60 \\
\text { anos. Os valores de EMAP foram significativamente maiores nos } \\
\text { homens do que nas mulheres, em ambas as mãos. }\end{array}$ \\
\hline Bielemann et al. ${ }^{28}$ & $\begin{array}{l}\text { Indivíduos saudáveis } \\
\mathrm{n}=3485 \\
\text { Média da idade }=33 \text { anos }\end{array}$ & $\begin{array}{l}\text { Homens: } 24,2 \pm 4,2 \mathrm{~mm} \\
\text { Mulheres: } 19,4 \pm 3,9 \mathrm{~mm}\end{array}$ & $\begin{array}{l}\text { O estudo não analisou a diferença da EMAP entre as mãos, } \\
\text { nem de acordo com a idade/ faixa etária. A EMAP foi maior nos } \\
\text { homens do que nas mulheres. Teve como objetivo verificar a } \\
\text { relação entre EMAP e massa magra. }\end{array}$ \\
\hline Lameu et al. ${ }^{30}$ & $\begin{array}{l}\text { Indivíduos saudáveis } \\
\mathrm{n}=421 \\
\text { Média da idade }=44 \pm 19 \text { anos }\end{array}$ & $\begin{array}{l}\text { Amostra total: } 11,5 \pm 2,7 \mathrm{~mm} \\
\text { Homens: } 12,5 \pm 2,8 \mathrm{~mm} \\
\text { Mulheres: } 10,5 \pm 2,3 \mathrm{~mm} \\
\text { Grupo > } 65 \text { anos: } \\
\text { Ambos os sexos: } 10,9 \pm 2,6 \mathrm{~mm} \\
\text { Homens: } 11,7 \pm 2,9 \mathrm{~mm} \\
\text { Mulheres: } 11,7 \pm 2,9 \mathrm{~mm}\end{array}$ & $\begin{array}{l}\text { Homens apresentaram valores superiores de EMAP. Foi verificada } \\
\text { redução significativa nos valores de EMAP quando comparados } \\
\text { indivíduos do grupo } 45-65 \text { anos e }>65 \text { anos, sendo } p<0,05 \text {. }\end{array}$ \\
\hline Freitas et al. ${ }^{36}$ & $\begin{array}{l}\text { Pacientes oncológicos } \\
\mathrm{n}=82 \\
\text { Média da idade }=49 \pm 16 \text { anos }\end{array}$ & $\begin{array}{l}\text { Amostra total: } 13,0 \pm 3,2 \mathrm{~mm} \\
\text { Homens: } 13,6 \mathrm{~mm} \\
\text { Mulheres: } 12,7 \mathrm{~mm}\end{array}$ & $\begin{array}{l}\text { A média da EMAP foi maior em homens do que em mulheres. } \\
\text { Foram verificados menores valores de EMAP em pacientes na } \\
\text { faixa etária dos 82-90 anos. }\end{array}$ \\
\hline Poziomyck et al. ${ }^{31}$ & $\begin{array}{l}\text { Pacientes oncológicos } \\
\mathrm{n}=74 \\
\text { Média da idade }=63 \pm 0 \text { anos }\end{array}$ & $\begin{array}{l}\text { MD: } 12,9 \pm 3,5 \mathrm{~mm} \\
\text { MND: } 12,0 \pm 3,6 \mathrm{~mm}\end{array}$ & $\begin{array}{l}\text { Não foi observada diferença entre os valores médios das medidas } \\
\text { da EMAP da MD e MND. }\end{array}$ \\
\hline De Oliveira et al. ${ }^{32}$ & $\begin{array}{l}\text { Pacientes em hemodiálise } \\
\mathrm{n}=143 \\
\text { Média da idade }=52 \pm 16 \text { anos }\end{array}$ & $\begin{array}{l}\text { Amostra total: } 11,8 \pm 1,6 \mathrm{~mm} \\
\text { Homens: } 12,3 \pm 1,5 \mathrm{~mm} \\
\text { Mulheres: } 11,1 \pm 1,5 \mathrm{~mm}\end{array}$ & $\begin{array}{l}\text { Foi verificada diferença significativa entre os valores médios da } \\
\text { EMAP de homens e mulheres }(p<0,0001) \text {. Não foi observada } \\
\text { diferença significativa nos valores de EMAP de acordo com a } \\
\text { idade, mas em homens acima dos } 60 \text { anos houve uma tendência } \\
\text { em reduzir os valores de EMAP. }\end{array}$ \\
\hline Pereira et al. ${ }^{33}$ & $\begin{array}{l}\text { Pacientes em hemodiálise } \\
\mathrm{n}=73 \\
\text { Média da idade }=52 \pm 17 \text { anos }\end{array}$ & $\begin{array}{l}\text { Total da amostra: } 10,0 \pm 4,5 \mathrm{~mm} \\
\text { Homens: } 10,8 \pm 4,9 \mathrm{~mm} \\
\text { Mulheres: } 9,0 \pm 3,7 \mathrm{~mm}\end{array}$ & $\begin{array}{l}\text { A EMAP não diferiu entres os sexos }(p=0,08) \text { e correlacionou-se } \\
\text { negativamente com a idade } \\
(r=-0,321 ; p<0,05)\end{array}$ \\
\hline Machado et al. ${ }^{29}$ & $\begin{array}{l}\text { Pacientes em hemodiálise } \\
\mathrm{n}=33 \\
\text { Média da idade }=68 \pm 11 \text { anos }\end{array}$ & $\begin{array}{l}\text { Total da amostra: } 15,9 \pm 3,7 \mathrm{~mm} \\
\text { Homens: } 17,4 \pm 3,4 \mathrm{~mm} \\
\text { Mulheres: } 14,1 \pm 3,3 \mathrm{~mm} \\
<60 \text { anos: } 19,4 \pm 4,1 \mathrm{~mm} \\
\geq 60 \text { anos: } 15,1 \pm 3,2 \mathrm{~mm}\end{array}$ & $\begin{array}{l}\text { O valor da EMAP mostrou-se superior na faixa etária }<60 \text { anos e } \\
\text { nos homens. }\end{array}$ \\
\hline Cobêro et al. ${ }^{34}$ & $\begin{array}{l}\text { Pacientes clínicos e cirúrgicos } \\
\text { hospitalizados } n=112 \\
\text { Média da idade }=53 \pm 17 \text { anos }\end{array}$ & Amostra total: $12,4 \pm 5,1 \mathrm{~mm}$ & $\begin{array}{l}\text { O estudo não analisou a diferença da EMAP entre as mãos, } \\
\text { nem de acordo com sexo ou idade/faixa etária. Teve como } \\
\text { objetivo verificar a associação entre a EMAP e indicadores } \\
\text { antropométricos. }\end{array}$ \\
\hline Bragagnolo et al..$^{35}$ & $\begin{array}{l}\text { Pacientes candidatos a } \\
\text { cirurgia de grande porte no } \\
\text { trato gastrointestinal } \\
n=87 \\
\text { Média da idade }=53 \pm 15 \text { anos }\end{array}$ & $\begin{array}{l}\text { MD: } 12,6 \pm 3,1 \mathrm{~mm} \\
\text { MND: } 12,2 \pm 2,9 \mathrm{~mm}\end{array}$ & $\begin{array}{l}\text { O estudo não analisou a diferença da EMAP entre as mãos, nem } \\
\text { de acordo com sexo ou idade/faixa etária. Teve como objetivo } \\
\text { verificar se a EMAP correlaciona-se bem com parâmetros } \\
\text { clínicos, antropométricos e bioquímicos. }\end{array}$ \\
\hline Andrade et al. ${ }^{22}$ & $\begin{array}{l}\text { Pacientes hospitalizados } \\
\text { portadores de doença valvar } \\
\text { mitral e/ou aórtica com } \\
\text { indicação cirúrgica } n=99 \\
\text { Média da idade }=50 \pm 16 \text { anos }\end{array}$ & Amostra total: $9,5 \pm 2,9 \mathrm{~mm}$ & $\begin{array}{l}\text { O estudo não analisou a diferença da EMAP entre as mãos, nem } \\
\text { de acordo com sexo ou idade/faixa etária. Teve como objetivo } \\
\text { comparar a medida da EMAP com parâmetros de avaliação } \\
\text { nutricional clássicos e avaliar se a EMAP é um bom índice } \\
\text { prognóstico em cirurgia cardíaca valvar. }\end{array}$ \\
\hline
\end{tabular}

EMAP: espessura do músculo adutor polegar; MD: mão dominante; MND: mão não dominante. 
No estudo de Freitas et al. ${ }^{36}$ com pacientes oncológicos e na investigação de De Oliveira et al. ${ }^{32}$ com pacientes em hemodiálise, foi verificada diferença significativa nos valores de EMAP quanto ao sexo, evidenciando maior reserva muscular encontrada em homens ${ }^{30,32}$, justificando a busca pelo estabelecimento de pontos de corte diferenciados entre homens e mulheres. Já o estudo de Pereira et al. ${ }^{33}$, com pacientes em hemodiálise, foi o único que não observou diferença quanto aos valores de EMAP em relação ao sexo.

Em relação à comparação entre a medida da EMAP de ambas as mãos, Caporossi et al. ${ }^{23}$, em um estudo com pacientes clínicos e cirúrgicos hospitalizados, observaram média da EMAP da mão direita superior à da mão esquerda $(p<0,001)$, embora tenham verificado correlação significativa $(r=0,84, p<0,001)$ entre a média da EMAP das duas mãos. No estudo de Poziomyck et al. ${ }^{31}$, não foi observada diferença entre os valores médios da EMAP entre as mãos. No presente estudo, também não se verificou diferença significativa da EMAP entre a MD e a MND. Portanto, os resultados sugerem que se possa obter a medida da EMAP em qualquer uma das mãos, fato este que apresenta relevância clínica, pois o paciente pode apresentar situações que restringem a avaliação da mão dominante como amputação, acesso venoso, curativo.

$\mathrm{Na}$ verificação da correlação entre EMAP e idade, no presente estudo observou-se correlação inversa, porém fraca. Pereira et al. ${ }^{33}$, também verificaram correlação inversa $(r=-0,321$ e $p<0,05)$. No estudo de Lameu et al. ${ }^{30}$, foi verificada redução significativa dos valores de EMAP quando comparado o grupo 45-65 anos com o grupo >65 anos, assim como no estudo de Freitas et al. ${ }^{36}$ foram observados menores valores de EMAP na faixa dos 82-90 anos. O estudo de De Oliveira et al. ${ }^{32}$ evidenciou uma tendência de redução dos valores de EMAP em homens acima dos 60 anos. Tais achados podem estar relacionados à redução da massa magra que ocorre com o envelhecimento ${ }^{37}$ e com a desnutrição ${ }^{35}$.

Como fatores limitantes do presente estudo, destaca-se a heterogeneidade dos pacientes idosos que se encontravam internados na Unidade Geriátrica do hospital, tanto relativa ao perfil clínico/cirúrgico (morbidades), quanto à capacidade funcional.

Cabe destacar que a EMAP tem sido descrita como um indicador de massa muscular e uma ferramenta útil na avaliação antropométrica ${ }^{38}$. Contudo, sua aplicação como método de triagem para risco nutricional merece estudos adicionais, especialmente em amostras com condições clínicas específicas e diferentes etnias, uma vez que os estudos são restritos à população brasileira.

Finalmente, conclui-se que, em idosos hospitalizados, a espessura do músculo adutor do polegar de ambas as mãos (dominante e não dominante) mostrou-se relacionada com sexo e faixa etária. No caso, a EMAP apresentou-se maior nos indivíduos do sexo masculino e nos indivíduos mais jovens (60-79 anos)

\section{REFERÊNCIAS}

1. Waitzberg DL, Caiaffa WT, Correia MITD. Hospital malnutrition: The Brazilian national survey (IBRANUTRI): A study of 4000 patients. Nutrition. 2001;17(7/8):573-80. https://doi.org/10.1016/ S0899-9007(01)00573-1

2. Santos CA, Firmino HH, Esmeraldo MLF, Alfenas RDCG, Rosa CDOB, Ribeiro AQ, Almeida LF, Amorim GP. Perfil nutricional e fatores associados à desnutrição e ao óbito em pacientes com indicação de terapia nutricional. Braspen J. 2017;32(1):30-5.

3. Fragas RFM, de Oliveira MC. Fatores de risco associados à desnutrição em idosos hospitalizados. Rev Nutr. 2016;29(3): 329-36. https://doi.org/10.1590/1678-98652016000300003

4. Valente da Silva HG, Santos SO, Silva NO, Ribeiro FD, Josua LL, B Moreira AS. Nutritional assessment associated with length of inpatients' hospital stay. Nutr Hosp. 2012;27(2):542-7.

5. Pfrimer K, Ferriolli E. Fatores que interferem no estado nutricional. In: Vitolo MR, organizador. Nutrição da gestação ao envelhecimento. 2a ed. Rio de Janeiro: Rubio; 2015. p. 410-3.

6. Volkert D. Malnutrition in older adults-urgent need for action: a plea for improving the nutritional situation of older adults. Gerontology. 2013;59(4):328-33. https://doi.org/10.1159/000346142

7. Sullivan D. The role of nutrition in increased morbidity and mortality. Clin Geriatr Med. 1995;11(4):661-74.

8. Verbrugghe $M$, Beeckman D, Van Hecke A, Vanderwee K, Van Herck K, Clays E, Bocquaert I, Dericke H, Geruden B, Verhaeghe S. Malnutrition and associated factors in nursing home residents: A cross-sectional, multi-centre study. Clin Nutr. 2013;32(3):438-43. https://doi.org/10.1016/j.clnu.2012.09.008

9. Gentile S, Lacroix O, Durand AC, Cretel E, Alazia M, Sambuc R, Bonin-Guillaume S. Malnutrition: a highly predictive risk factor of short-term mortality in elderly presenting to the emergency department. J Nutr Health Aging. 2013;17(4):290-4. https://doi. org/10.1007/s12603-012-0398-0

10. Santos VH, Rezende CHA de. Nutrição e envelhecimento. In: Freitas EV de, Py L, Cançado FAX, Doll J, Gorzoni ML, organizadores. Tratado de geriatria e gerontologia. $2^{a}$ ed. Rio de Janeiro: Guanabara Koogan; 2006. p. 930-41.

11. Olivares J, Ayala L, Salas-Salvadó J, Mu-iz MJ, Gamundí A, Martínez-Indart L, Masmiquel LL. Assessment of risk factors and test performance on malnutrition prevalence at admission using four different screening tools. Nutr Hosp. 2014;29(3):674-80.

12. Vellas B, Guigoz Y, Garry P, Nourhashemi F, Bennahum D, Lauque S, Albarede JL. The Mini Nutritional Assessment (MNA) and its use in grading the nutritional state of elderly patients. Nutrition. 1999;15(2):166-22. https://doi.org/10.1016/S08999007(98)00171-3

13. Vellas B, Villars H, Abellan M, Soto $Y$, Rolland Y, Y G. Overview of the $M N A \AA$-Its history and challenges. J Nutr Heal Aging. $2006 ; 10(6): 456-65$. 
14. Guigoz Y. The Mini Nutritional Assessment (MNA) review of the literature-What does it tell us? J Nutr Health Aging. 2006;10(6): 466-85; discussion 485-7.

15. Haverkort EB, Binnekade JM, de Haan RJ, Schueren MAEB. Handgrip strength by dynamometry does not identify malnutrition in individual preoperative outpatients. Clin Nutr. 2012;31(5): 647-51. https://doi.org/10.1016/j.clnu.2012.01.010

16. Lenardt MH, Grden CRB, de Sousa JAV, Reche P M, Betiolli SE, Ribeiro DKMN. Fatores associados à diminuição de força de preensão manual em idosos longevos. Rev Esc Enferm USP. 2014;48(6): 1006-12. https://doi.org/10.1590/S0080-623420140000700007

17. Martin FG, Nebuloni CC, Najas, MS. Correlação entre estado nutricional e força de preensão palmar em idosos. Rev Bras Geriatr Gerontol. 2012;15(3):493-504. https://doi.org/10.1590/S180998232012000300010

18. Tagliapietra BL, Vaz TL, Schuch NJ, Margutti KM. Preditores para diagnóstico de sarcopenia, estado nutricional e atividade física de idosas institucionalizadas e não institucionalizadas. Discipl Sci Cienc Saúde. 2016;17(1):53-62.

19. Pereira CA, Moreno JG, El Kik RM. Utilização da espessura do músculo adutor do polegar na avaliação nutricional. Cienc Saúde. 2014;7(2):109-14. https://doi.org/10.15448/1983$652 X .2014 .2 .16626$

20. Lameu E. Músculo adutor do polegar. In: Lameu E, organizador. Clínica nutricional. Rio de Janeiro: Revinter; 2005. p. 189-95.

21. Lameu EB, Gerude MF, Corrêa RC, Lima KA. Adductor pollicis muscle: a new anthropometric parameter. Rev Hosp Clin Fac Med São Paulo. 2004;59(2):57-62. https://doi.org/10.1590/S004187812004000200002

22. Andrade FN, Lameu EB, Luiz RR. Musculatura Adutora do polegar: um novo índice prognóstico em cirurgia cardíaca valvar. Rev SOCERJ. 2005;18(5):384-91.

23. Caporossi FS, Caporossi C, Dock-Nascimento DB, de AguilarNascimento JE. Measurement of the thickness of the adductor pollicis muscle as a predictor of outcome in critically ill patients. Nutr Hosp. 2012;27(2):490-5.

24. Fleiss JL, Cohen J. The equivalence of weighted Kappa and the intraclass correlation coeficient as a measure of reliability. Education Psychol Measurem. 1973;33(3):613-9. https://doi. org/10.1177/001316447303300309

25. Callegari-Jacques, SM. Correlação linear simples. Bioestatística: princípios e aplicações. São Paulo: Artmed; 2003.

26. Melo CYSV de, Silva SA da. Músculo adutor do polegar como preditor de desnutrição em pacientes cirúrgicos. ABCD Arq Bras Cir Dig. 2014;27(1):13-7.

27. Gonzalez MC, Duarte RRP, Budziareck MB. Adductor pollicis muscle: reference values of its thickness in a healthy population. Clin Nutr. 2010;29(2):268-71. https://doi.org/10.1016/j.clnu.2009. 08.012
28. Bielemann RM, Horta BL, Orlandi SP, Barbosa-Silva TG, Gonzalez MC, Assunção MC, Gigante DP. Is adductor pollicis muscle thickness a good predictor of lean mass in adults? Clin Nutr. 2016;35(5): 1073-7. https://doi.org/10.1016/j.clnu.2015.07.022

29. Machado AD, Rissotto CM, Martins CTB, Bazanelli AP. Associação entre o consumo energético e proteico e a espessura do músculo adutor do polegar em pacientes em hemodiálise. Cienc Saúde; 2017;10(1):3-9. https://doi.org/10.15448/1983$652 X .2017 .1 .24111$

30. Lameu EB, Gerude MF, Campos AC, Luiz RR. The thickness of the adductor pollicis muscle reflects the muscle compartment and may be used as a new anthropometric parameter for nutritional assessment. Curr Opin Clin Nutr Metab Care. 2004; 7(3):293-301. https://doi.org/10.1097/00075197-200405000-00009

31. Poziomyck AK, Weston AC, Lameu EB, Cassol OS, Coelho LJ, Moreira LF. Preoperative nutritional assessment and prognosis in patients with foregut tumors. Nutr Cancer. 2012;64(8):1174-81. https://doi.org/10.1080/01635581.2012.721157

32. De Oliveira CMC, Kubrusly M, Mota RS, Choukroun G, Neto JB, Da Silva CAB. Adductor pollicis muscle thickness: a promising anthropometric parameter for patients with chronic renal failure. J Ren Nutr. 2012;22(3):307-16. https://doi.org/10.1053/j. jrn.2011.07.006

33. Pereira RA, Caetano AL, Cuppari L, Kamimura MA. Adductor pollicis muscle thickness as a predictor of handgrip strength in hemodialysis patients. J Bras Nefrol. 2013;35(3):177-84. https:// doi.org/10.5935/0101-2800.20130029

34. Cobêro FE, Gomes MCB, Silva AP, Bernardi JLD, McLellan KCP. A medida do músculo adutor do polegar está associada com indicadores antropométricos de avaliação de massa magra e de massa gorda em pacientes hospitalizados. Nutrire. 2012;37(2): 174-82. https://doi.org/10.4322/nutrire.2012.014

35. Bragagnolo R, Caporossi FS, Dock-Nascimento DB, AguilarNascimento JE de. Espessura do músculo adutor do polegar: um método rápido e confiável na avaliação nutricional de pacientes cirúrgicos. Rev Col Bras Cir. 2009;36(5):371-6. https://doi. org/10.1590/S0100-69912009000500003

36. Freitas BJSA, Mesquita LC, Teive N de JV, Souza SR. Antropometria clássica e músculo adutor do polegar na determinação do prognóstico nutricional em pacientes oncológicos. Rev Bras Cancerol. 2010;56(4):415-22.

37. França AP, Pivi GAK. Alterações orgânicas, fisiológicas e metabólicas do processo de envelhecimento e seus reflexos na nutrição do idoso. In: Silva M de L do N, Maruci M de FN, Roediger M de A, organizadores. Tratado de nutrição em gerontologia. Baruri, SP: Manole; 2016. p. 49-61.

38. Gonzalez MC, Pureza Duarte RR, Orlandi SP, Bielemann RM, Barbosa-Silva TG. Adductor pollicis muscle: A study about its use as a nutritional parameter in surgical patients. Clin Nutr. 2015;34(5):1025-9. https://doi.org/10.1016/j.clnu.2014.11.006 